TRANSACTIONS OF THE

AMERICAN MATHEMATICAL SOCIETY

Volume 362, Number 6, June 2010, Pages 3107-3123

S 0002-9947(09)04865-X

Article electronically published on December 22, 2009

\title{
CODIMENSION GROWTH OF SPECIAL SIMPLE JORDAN ALGEBRAS
}

\author{
ANTONIO GIAMBRUNO AND MIKHAIL ZAICEV
}

\begin{abstract}
Let $R$ be a special simple Jordan algebra over a field of characteristic zero. We exhibit a noncommutative Jordan polynomial $f$ multialternating on disjoint sets of variables which is not a polynomial identity of $R$. We then study the growth of the polynomial identities of the Jordan algebra $R$ through an analysis of its sequence of Jordan codimensions. By exploiting the basic properties of the polynomial $f$, we are able to compute the exponential rate of growth of the sequence of Jordan codimensions of $R$ and prove that it equals the dimension of the Jordan algebra over its center. We also show that for any finite dimensional special Jordan algebra, such an exponential rate of growth cannot be strictly between 1 and 2 .
\end{abstract}

\section{INTRODUCTION}

Let $F$ be a field of characteristic zero and $A$ a not necessarily associative algebra over $F$. The study of the polynomial identities satisfied by $A$ has been carried out in an effective way through the representation theory of the symmetric group. The combination of algebraic and analytical methods in this theory leads to significant results (see for instance [3], 4], 11, 19]).

The identities of an algebra $A$ form a $T$-ideal $\operatorname{Id}(A)$ of the free nonassociative algebra $F\{X\}$, and one associates to $\operatorname{Id}(A)$ an integral sequence $c_{n}(A), n=1,2, \ldots$, called the sequence of codimensions of $A$. Recall that if $P_{n}$ is the space of multilinear polynomials in the variables $x_{1}, \ldots, x_{n}$, then $c_{n}(A)=\frac{P_{n}}{P_{n} \cap I d(A)}$. Such a sequence measures in some way the polynomial relations vanishing in an algebra $A$ and in general has overexponential growth. In order to study such a sequence, several methods have been developed over the years ([11, 19]) and the most significant results have been obtained when $c_{n}(A)$ is exponentially bounded.

In this setting a celebrated theorem of Regev ([21]) states that any associative algebra satisfying a nontrivial polynomial identity (PI-algebra) has the sequence of the codimensions exponentially bounded. Moreover the class of nonassociative algebras sharing such a property is quite wide and includes finite dimensional algebras ([1]), infinite dimensional simple Lie algebras of Cartan type ([17]), affine Kac-Moody algebras $(22])$, etc. In case $c_{n}(A)$ is exponentially bounded, one

Received by the editors April 16, 2008.

2000 Mathematics Subject Classification. Primary 17C05, 16P90; Secondary 16R10.

Key words and phrases. Polynomial identity, special Jordan algebra, codimensions, exponential growth.

The first author was partially supported by MIUR of Italy.

The second author was partially supported by RFBR grant No. 06-01-00485 and SSC5666.2006.1. 
can construct the bounded sequence $\sqrt[n]{c_{n}(A)}, n=1,2, \ldots$, and ask if $\exp (A)=$ $\lim _{n \rightarrow \infty} \sqrt[n]{c_{n}(A)}$ exists; $\exp (A)$ is called the PI-exponent of the algebra $A$.

In [10] it was proved that for any associative PI-algebra $A, \exp (A)$ exists and is an integer. In the case of finite dimensional Lie algebras, in 23] it was shown that the PI-exponent also exists and is an integer. The same conclusion was achieved in [12] for the Jordan algebra $M_{k}(F)$ of $k \times k$ matrices and in [9] for some special simple Jordan algebras of small dimension. These results about the integrality of the PI-exponent are quite surprising since in [7] the authors constructed, for any real number $\alpha>1$, a nonassociative algebra whose exponential rate of growth of the codimensions equals $\alpha$.

The main purpose of this paper is to prove the existence of the PI-exponent for any finite dimensional special simple Jordan algebra. Recall that if $F$ is algebraically closed, then any such algebra is isomorphic to one of the following: 1) $M_{k}(F)$ with the Jordan product $\left.a \circ b=a b+b a, 2\right)$ the Jordan algebra $\left(M_{k}(F), t\right)^{+}$ of $k \times k$ symmetric matrices under the transpose involution, 3) the Jordan algebra $\left(M_{k}(F), s\right)^{+}$of $k \times k$ symmetric matrices under the symplectic involution, 4) the Jordan algebra defined by a nondegenerate symmetric bilinear form on a $k$-dimensional vector space. The main result proved here is the following.

Theorem. Let $A$ be a finite dimensional special simple Jordan algebra over a field of characteristic zero. Then $\exp (A)$ exists and equals the dimension of $A$ over its center.

Of independent interest is also a construction for any such algebra, of a multialternating Jordan polynomial in $k \cdot \operatorname{dim} A$ variables for any $k \geq 1$, which is not an identity of $A$. We refer the reader to [14] for the basic properties of Jordan algebras.

The last section of the paper is devoted to finite dimensional special Jordan algebras and slow exponential growth of the codimensions.

In [5] it was proved that if $A$ is a finite dimensional algebra, $\operatorname{dim} A=d$, then either $c_{n}(A)$ is polynomially bounded or $c_{n}(A)>\frac{1}{n^{2}} 2^{\frac{n}{3 d^{2}}}$ for $n$ large enough. Moreover by [7, given any real numbers $1<\alpha<\beta<2$ there exists a finite dimensional algebra $B$ such that $\alpha<\exp (B)<\beta$.

Despite these results, the exponential rate of growth of the codimensions cannot be less than 2 for a wide class of algebras. In fact, if $A$ is either an associative algebra ([16]) or a Lie algebra $([18])$, then the asymptotic inequality $c_{n}(A)<2^{n}$ implies $c_{n}(A) \leq f(n)$ for some polynomial $f$. The same phenomenon occurs in the case of two and three dimensional nonassociative algebras (6, 6] ) and finite dimensional Lie superalgebras $([24)$. In the last section we show that finite dimensional special Jordan algebras also cannot have exponential codimension growth strictly between 1 and 2 .

Recall that given an associative algebra $A$, the Lie commutator $[a, b]=a b-b a$ defines a structure of a Lie algebra on $A$. Similarly the circle operation $a \circ b=a b+b a$ defines a structure of a Jordan algebra. Throughout we shall use the left-normed notation on Lie and Jordan monomials; i.e., $\left[x_{1}, \ldots, x_{n}\right]$ denotes $\left[\left[x_{1}, x_{2}\right], \ldots, x_{n}\right]$ and similarly for Jordan products $x_{1} \circ \cdots \circ x_{n}=\left(\left(x_{1} \circ x_{2}\right) \cdots\right) \circ x_{n}$.

Let $X=\left\{x_{1}, x_{2}, \ldots\right\}$ be a countable set. We denote by $F\langle X\rangle$ the free associative algebra on $X$ over $F$ and by $F^{J}\langle X\rangle$ and $F^{L}\langle X\rangle$ the free special Jordan algebra and the free Lie algebra on $X$, respectively. Throughout we shall tacitly assume that 
$F^{J}\langle X\rangle$ and $F^{L}\langle X\rangle$ are canonically embedded in $F\langle X\rangle$. Also, we shall use other symbols such as $y, z, x_{i}^{j}$ for extra new indeterminates in $F\langle X\rangle$.

\section{Multialternating Lie POlynomials And Symmetric matrices}

Let $A$ be any associative algebra and $V \subseteq A$ a subspace. Recall that a polynomial $f\left(x_{1}, \ldots, x_{t}\right) \in F\langle X\rangle$ is an identity of $V$ if $f\left(v_{1}, \ldots, v_{t}\right)=0$ for any $v_{1}, \ldots, v_{t} \in V$. Also, a polynomial $f \in F^{L}\langle X\rangle$ is called a Lie polynomial and $f \in F^{J}\langle X\rangle$ is a Jordan polynomial. There is a connection between Lie and Jordan polynomials given in the following.

Lemma 1. Any multilinear Lie polynomial of odd degree is a Jordan polynomial.

Proof. Since any Lie polynomial is a sum of left-normed monomials, the proof easily follows from the equality

$$
[a, b, c]=a \circ(c \circ b)-(a \circ c) \circ b .
$$

Lemma 2. Let $f=f\left(x_{1}, \ldots, x_{m}, y_{1}, \ldots, y_{k}\right)$ be a multilinear Lie polynomial alternating on $x_{1}, \ldots, x_{m}$. Then, for $v, z \in X$, the polynomial

$$
g=\sum_{i=1}^{m} f\left(x_{1}, \ldots, x_{i-1},\left[x_{i}, v, z\right], x_{i+1}, \ldots, x_{m}, y_{1}, \ldots, y_{k}\right)
$$

is also alternating on $x_{1}, \ldots, x_{m}$.

Proof. Clearly it is enough to check that $f$ is alternating on each pair $x_{\alpha}, x_{\beta}$, $1 \leq \alpha<\beta \leq m$. For short let $\alpha=1$ and $\beta=2$. Since the polynomial

$$
\sum_{i=3}^{m} f\left(x_{1}, \ldots,\left[x_{i}, v, z\right], \ldots, x_{m}, y_{1}, \ldots, y_{k}\right)
$$

is alternating on $x_{1}$ and $x_{2}$, it is enough to check that

$$
g^{\prime}=f\left(\left[x_{1}, v, z\right], x_{2}, \ldots, x_{m}, y_{1}, \ldots, y_{k}\right)+f\left(x_{1},\left[x_{2}, v, z\right], x_{3}, \ldots, x_{m}, y_{1}, \ldots, y_{k}\right)
$$

is alternating on $x_{1}$ and $x_{2}$. But

$$
\begin{aligned}
& g^{\prime}\left(x_{1}, x_{2}, \ldots\right)+g^{\prime}\left(x_{2}, x_{1}, \ldots\right)=f\left(\left[x_{1}, v, z\right], x_{2}, \ldots\right) \\
& \quad+f\left(x_{1},\left[x_{2}, v, z\right], \ldots\right)+f\left(\left[x_{2}, v, z\right], x_{1}, \ldots\right)+f\left(x_{2},\left[x_{1}, v, z\right], \ldots\right) \\
& \quad=f\left(\left[x_{1}, v, z\right], x_{2}, \ldots\right)-f\left(\left[x_{2}, v, z\right], x_{1}, \ldots\right) \\
& \quad+f\left(\left[x_{2}, v, z\right], x_{1}, \ldots\right)-f\left(\left[x_{1}, v, z\right], x_{2}, \ldots\right) \equiv 0
\end{aligned}
$$

since $f(x, y, \ldots)=-f(y, x, \ldots)$.

Throughout we let $R=M_{q}(F)$ denote the algebra of $q \times q$ matrices over a field $F$ of characteristic zero and we let $*$ denote either the transpose involution denoted $*=t$ or the symplectic involution denoted $*=s$ on $R$. Since we are interested in simple Jordan algebras, we shall also assume that $q \geq 3$. Define

$$
R^{(+)}=\left\{a \in R \mid a^{*}=a\right\}, \quad R^{(-)}=\left\{a \in R \mid a^{*}=-a\right\} .
$$

Then $R^{(+)}$is a Jordan algebra, $R^{(-)}$is a Lie algebra and $R=R^{(+)} \oplus R^{(-)}$is a vector space decomposition. Let $L=s l_{q}(F) \subseteq R$ be the Lie algebra of $q \times q$ 
traceless matrices. Then $L^{*}=L$ and we have

$$
L=L^{(+)} \oplus L^{(-)},
$$

where $L^{(+)}=s l_{q}(F) \cap R^{(+)}$and $L^{(-)}=R^{(-)}$.

Recall that, given $a \in R$, the adjoint $\operatorname{map}$ ad $a: R \rightarrow R$ is the left Lie multiplication ad $a: b \rightarrow[a, b]$. It is readily seen that if $a \in L^{(+)}$is symmetric, then ad $a\left(L^{(+)}\right) \subseteq L^{(-)}$and ad $a\left(L^{(-)}\right) \subseteq L^{(+)}$. Hence, for any $a, b \in L^{(+)}$, the product $(\operatorname{ad} a)(\operatorname{ad} b)$ preserves $L^{(+)}$and $L^{(-)}$. We shall denote by $\operatorname{tr}(\operatorname{ad} a \operatorname{ad} b)$ the trace of the linear transformation ad $a$ ad $b$ on $L^{(+)}$.

In what follows, in order to simplify the notation, we shall often write $f=$ $f\left(x_{1}, \ldots, x_{m}, y_{1}, \ldots, y_{n}\right)=f\left(x_{1}, \ldots, x_{m}, Y\right)$, where $Y=\left\{y_{1}, \ldots, y_{n}\right\}$.

Lemma 3. Let $Y=Y_{0} \cup Y_{1} \cup \cdots \cup Y_{r} \subseteq X$ be a disjoint union with $r \geq 0$ and $Y_{0}$ eventually empty. Let $f=f\left(x_{1}, \ldots, x_{m}, Y\right)$ be a multilinear Lie polynomial alternating on each $Y_{i}, 1 \leq i \leq r$, and on $x_{1}, \ldots, x_{m}$, where $m=\operatorname{dim} L^{(+)}$. Then, for any $k \geq 1$ and for any $v_{1}, z_{1}, \ldots, v_{k}, z_{k} \in X$, there exists a multilinear Lie polynomial

$$
g=g\left(x_{1}, \ldots, x_{m}, v_{1}, z_{1}, \ldots, v_{k}, z_{k}, Y\right)
$$

such that, for any evaluation $\varphi: X \rightarrow L^{(+)}, \varphi\left(x_{i}\right)=\bar{x}_{i}, 1 \leq i \leq m, \varphi\left(v_{j}\right)=\bar{v}_{j}$, $\varphi\left(z_{j}\right)=\bar{z}_{j}, 1 \leq j \leq k, \varphi(y)=\bar{y}$, for $y \in Y$, we have

$$
\begin{aligned}
& \varphi(g)=g\left(\bar{x}_{1}, \ldots, \ldots, \bar{x}_{m}, \bar{v}_{1}, \bar{z}_{1}, \ldots, \bar{v}_{k}, \bar{z}_{k}, \bar{Y}\right) \\
& \quad=\operatorname{tr}\left(\operatorname{ad} \bar{v}_{1} \operatorname{ad} \bar{z}_{1}\right) \cdots \operatorname{tr}\left(\operatorname{ad} \bar{v}_{k} \operatorname{ad} \bar{z}_{k}\right) f\left(\bar{x}_{1}, \ldots, \bar{x}_{m}, \bar{Y}\right) .
\end{aligned}
$$

Moreover $g$ is alternating on each set $Y_{i}, 1 \leq i \leq r$, and on $x_{1}, \ldots, x_{m}$.

Proof. The proof is by induction of $k$. Suppose first that $k=1$. Then we define

$$
g=g\left(x_{1}, \ldots, \ldots, x_{m}, v, z, Y\right)=\sum_{i=1}^{m} f\left(x_{1}, \ldots,\left[x_{i}, v, z\right], \ldots, x_{m}, Y\right) .
$$

Clearly $g$ is alternating on each set $Y_{i}, 1 \leq i \leq r$ and, by Lemma 2, is also alternating on $x_{1}, \ldots, x_{m}$. Consider the evaluation $\varphi: X \rightarrow L^{(+)}$such that $\varphi\left(x_{i}\right)=$ $\bar{x}_{i}, 1 \leq i \leq m, \varphi(v)=\bar{v}, \varphi(z)=\bar{z}, \varphi(y)=\bar{y}$, for $y \in Y$. If the elements $\bar{x}_{1}, \ldots, \ldots, \bar{x}_{m}$ are linearly dependent over $F$, then, since $f$ and $g$ are alternating on $x_{1}, \ldots, x_{m}$, it follows that $\varphi(f)=\varphi(g)=0$ and there is nothing to prove.

Suppose then that $\bar{x}_{1}, \ldots, \bar{x}_{m}$ are linearly independent over $F$. Since $\operatorname{dim} L^{(+)}=$ $m$, they form a basis of $L^{(+)}$. Hence, for all $i=1, \ldots, m$, we can write

$$
\left[\bar{x}_{i}, \bar{v}, \bar{z}\right]=\alpha_{i i} \bar{x}_{i}+\sum_{j \neq i} \alpha_{i j} \bar{x}_{j}
$$

for some scalars $\alpha_{i j} \in F$. Since $f$ is alternating on $x_{1}, \ldots, x_{m}$, we have that

$$
f\left(\bar{x}_{1}, \ldots,\left[\bar{x}_{i}, \bar{v}, \bar{z}\right], \ldots, \bar{x}_{m}, \bar{Y}\right)=\alpha_{i i} f\left(\bar{x}_{1}, \ldots, \bar{x}_{i}, \ldots, \bar{x}_{m}, \bar{Y}\right) .
$$

It follows that

$$
g\left(\bar{x}_{1}, \ldots, \bar{x}_{m}, \bar{v}, \bar{z}, \bar{Y}\right)=\left(\alpha_{11}+\cdots+\alpha_{m m}\right) f\left(\bar{x}_{1}, \ldots, \bar{x}_{m}, \bar{Y}\right),
$$

and, since $\alpha_{11}+\cdots+\alpha_{m m}=\operatorname{tr}(\operatorname{ad} \bar{v}$ ad $\bar{z})$, the lemma is proved in case $k=1$.

Now let $k>1$ and suppose that we have constructed a multilinear Lie polynomial $g=g\left(x_{1}, \ldots, \ldots, x_{m}, v_{1}, z_{1}, \ldots, v_{k-1}, z_{k-1}, Y\right)$ satisfying the conclusion of the lemma. We then write $g=g\left(x_{1}, \ldots, \ldots, x_{m}, Y^{\prime}\right)$, where $Y^{\prime}=Y_{0}^{\prime} \cup Y_{1} \cup \cdots \cup Y_{r}$ and 
$Y_{0}^{\prime}=Y_{0} \cup\left\{v_{1}, z_{1}, \ldots, v_{k-1}, z_{k-1}\right\}$. If we now apply to $g$ the same arguments as in the case $k=1$, we obtain a polynomial satisfying the conclusion of the lemma.

The following remark holds.

Remark 1. If $q \geq 3$, then $L^{(+)}$is an irreducible $L^{(-)}$-module under the adjoint action.

Proof. Suppose first that $L^{(-)}$is a simple algebra. If $M$ is a proper $L^{(-)}$-submodule of $L^{(+)}$, then $[M, M]$ is an ideal of $L^{(-)}$. By the simplicity of $L^{(-)}$either $[M, M]=$ $L^{(-)}$or $[M, M]=0$.

Suppose first that $[M, M]=L^{(-)}$. Since $[M, M] \oplus M$ is an $L^{(-)}$-module and $\left[L^{(+)},[M, M]\right] \subseteq\left[\left[L^{(+)}, M\right], M\right] \subseteq\left[L^{(-)}, M\right] \subseteq M,\left[L^{(+)}, M\right] \subseteq L^{(-)}=[M, M]$, we conclude that $[M, M] \oplus M$ is a proper ideal of $L=s l_{q}(F)$, a contradiction.

In case $[M, M]=0$, we consider the decomposition $L^{(+)}=M \oplus N$, where $M$ and $N$ are $L^{(-)}$-submodules. By the same arguments as above we have that $[N, N]=0$. If we denote $L_{0}=L^{(-)}, L_{1}=M$ and $L_{2}=N$, then the decomposition $L=L_{0} \oplus L_{1} \oplus L_{2}$ is a $\mathbb{Z}_{3}$-grading of $L=s l_{q}(F)$. Now, it is well known that, up to isomorphism of graded algebras, any $\mathbb{Z}_{3}$-grading of $L=s l_{q}(F)$ is induced by a grading of $R=M_{q}(F)$; i.e., there exists a $\mathbb{Z}_{3}$-grading of $R, R=R_{0} \oplus R_{1} \oplus R_{2}$ such that $L_{1}=R_{1}, L_{2}=R_{2}$ and $L_{0}=L \cap R_{0}$ ([2]). On the other hand any $\mathbb{Z}_{3}$-grading of $M_{q}(F)$ is elementary ([1]) and $R_{0}$ is a semisimple not simple associative algebra. It follows that $R_{0} \cap s l_{q}(F)=L_{0}=L^{(-)}$cannot be simple and this is a contradiction. Thus $L^{(+)}$is an irreducible $L^{(-)}$-module and we are done.

Suppose now that $L^{(-)}$is not simple. Then by [13, $*=t$ is the transpose involution and $q=4$. In this case a direct computation shows that $L^{(+)}$is an irreducible $L^{(-)}$-module.

Definition 1. $\beta: L^{(+)} \times L^{(+)} \rightarrow F$ is the bilinear form defined by $\beta(a, b)=$ $\operatorname{tr}(\operatorname{ad} a \operatorname{ad} b)$, for $a, b \in L^{(+)}$.

Next we need to exploit some of the properties of the bilinear form $\beta$.

Lemma 4. The bilinear form $\beta$ is nondegenerate on $L^{(+)}=s l_{q}(F)^{(+)}$.

Proof. Denote by $I=\left\{a \in L^{(+)} \mid \beta\left(a, L^{(+)}\right)=0\right\}$ the (left) kernel of $\beta$. Notice that, since $\operatorname{ad}[a, b]=[\operatorname{ad} a, \operatorname{ad} b]$, the form $\beta$ satisfies the equality

$$
\beta([a, c], b)=\beta(a,[c, b]),
$$

for all $a, b \in L^{(+)}$and $c \in L^{(-)}$. If we consider the adjoint action of $L^{(-)}$on $L$, then $L^{(+)}$is an $L^{(-)}$-submodule. Now, the equality (2) implies that $\left[I, L^{(-)}\right] \subseteq I$, i.e., that $I$ is an $L^{(-)}$-submodule of $L^{(+)}$. By Remark 1 we have that either $I=0$ or $I=L^{(+)}$and, in order to complete the proof of the lemma, it is enough to check that $I \neq L^{(+)}$, i.e., that $\beta$ is nonzero.

Suppose first that $*$ is the symplectic involution on $M_{q}(F)$. Then $q=2 p$ is even and $*$ is given by

$$
\left(\begin{array}{cc}
A & B \\
C & D
\end{array}\right)^{*}=\left(\begin{array}{cc}
D^{t} & -B^{t} \\
-C^{t} & A^{t}
\end{array}\right),
$$

where $A, B, C, D$ are $p \times p$ matrices and $t$ denotes the transpose involution. The matrix $a=e_{11}+e_{p+1, p+1}$ is symmetric, and we let $\varphi=(\operatorname{ad} a)^{2}$. Since $\varphi^{2}=\varphi$, then $\varphi$ is a diagonalizable linear transformation of $L=s l_{q}(F)$ with eigenvalues 0 and 1. Hence the restriction of $\varphi$ to $L^{(+)}$is also diagonalizable with possibly the same 
eigenvalues. Since $\varphi\left(L^{(+)}\right) \neq 0$, we conclude that $\operatorname{tr} \varphi \neq 0$ and, so, $\beta(a, a) \neq 0$. This completes the proof in case $*$ is symplectic.

In case $*$ is the transpose involution, we choose $a=e_{11}+e_{22}$. Then, since $q \geq 3$, $\varphi=(\operatorname{ad} a)^{2}$ is a nonzero diagonalizable linear transformation of $L$ with eigenvalues 0 and 1. As above, since the restriction of $\varphi$ to $L^{(+)}$is nonzero, we conclude that $\beta(a, a)=\operatorname{tr} \varphi \neq 0$.

As an immediate consequence of the above lemma we have that, given any linearly independent elements $a_{1}, \ldots, a_{m} \in L^{(+)}$with $m=\operatorname{dim} L^{(+)}$, then

$$
\operatorname{det}\left(\beta_{i j}\right) \neq 0 \text {, }
$$

where $\beta_{i j}=\beta\left(a_{i}, a_{j}\right), 1 \leq i, j \leq m$. We shall use the above property in order to construct alternating polynomials nonvanishing in $L^{(+)}$and $R^{(+)}$.

Lemma 5. Let $f=f\left(x_{1}, \ldots, x_{m}, y_{1}, \ldots, y_{r}\right)$ be a multilinear Lie polynomial alternating on $x_{1}, \ldots, x_{m}$, where $m=\operatorname{dim} L^{(+)}$. Then, for any $k \geq 1$, there exists a multilinear Lie polynomial

$$
g_{k}\left(x_{1}, \ldots, x_{m}, v_{1}^{(1)}, z_{1}^{(1)}, \ldots, v_{m}^{(1)}, z_{m}^{(1)}, \ldots, v_{1}^{(k)}, z_{1}^{(k)}, \ldots, v_{m}^{(k)}, z_{m}^{(k)}, y_{1}, \ldots, y_{r}\right)
$$

satisfying the following conditions:

1) $g_{k}$ is alternating on each of the sets $\left\{x_{1}, \ldots, x_{m}\right\},\left\{v_{1}^{(i)}, \ldots, v_{m}^{(i)}\right\}$, and $\left\{z_{1}^{(i)}, \ldots, z_{m}^{(i)}\right\}, \quad 1 \leq i \leq k$

2) for any evaluation $\varphi: X \rightarrow L^{(+)}$, such that $\varphi\left(x_{i}\right)=\bar{x}_{i}, \varphi\left(y_{i}\right)=\bar{y}_{i}$, $\varphi\left(v_{i}^{(j)}\right)=\bar{v}_{i}^{(j)}, \varphi\left(z_{i}^{(j)}\right)=\bar{z}_{i}^{(j)}$, we have

$$
\varphi\left(g_{k}\right)=\left(\operatorname{det} \bar{\beta}_{1}\right) \cdots\left(\operatorname{det} \bar{\beta}_{k}\right) f\left(\bar{x}_{1}, \ldots, \bar{x}_{m}, \bar{y}_{1}, \ldots, \bar{y}_{r}\right),
$$

$$
\begin{aligned}
& \text { where } \\
& \bar{\beta}_{s}=\left(\begin{array}{ccc}
\beta\left(\bar{v}_{1}^{(s)}, \bar{z}_{1}^{(s)}\right) & \cdots & \beta\left(\bar{v}_{1}^{(s)}, \bar{z}_{m}^{(s)}\right) \\
\vdots & & \vdots \\
\beta\left(\bar{v}_{m}^{(s)}, \bar{z}_{1}^{(s)}\right) & \cdots & \beta\left(\bar{v}_{m}^{(s)}, \bar{z}_{m}^{(s)}\right)
\end{array}\right), \\
& s=1, \ldots, k .
\end{aligned}
$$

Proof. Suppose first that $k=1$ and write $Y=\left\{y_{1}, \ldots, y_{r}\right\}$. By Lemma 3 there exists a multilinear Lie polynomial

$$
g=g\left(x_{1}, \ldots, x_{m}, v_{1}^{(1)}, z_{1}^{(1)}, \ldots, v_{m}^{(1)}, z_{m}^{(1)}, Y\right)
$$

such that

$$
\begin{aligned}
& g\left(\bar{x}_{1}, \ldots, \bar{x}_{m}, \bar{v}_{1}^{(1)}, \bar{z}_{1}^{(1)}, \ldots, \bar{v}_{m}^{(1)}, \bar{z}_{m}^{(1)}, \bar{Y}\right) \\
& \quad=\operatorname{tr}\left(\operatorname{ad} \bar{v}_{1}^{(1)} \operatorname{ad} \bar{z}_{1}^{(1)}\right) \cdots \operatorname{tr}\left(\operatorname{ad} \bar{v}_{m}^{(1)} \operatorname{ad} \bar{z}_{m}^{(1)}\right) f\left(\bar{x}_{1}, \ldots, \bar{x}_{m}, \bar{Y}\right) .
\end{aligned}
$$

Now, for any $\sigma, \tau \in S_{m}$, we define the polynomial

$$
\begin{aligned}
& g_{\sigma, \tau}=g_{\sigma, \tau}\left(x_{1}, \ldots, x_{m}, v_{1}^{(1)}, z_{1}^{(1)}, \ldots, v_{m}^{(1)}, z_{m}^{(1)}, Y\right) \\
& \quad=g\left(x_{1}, \ldots, x_{m}, v_{\sigma(1)}^{(1)}, z_{\tau(1)}^{(1)}, \ldots, v_{\sigma(m)}^{(1)}, z_{\tau(m)}^{(1)}, Y\right)
\end{aligned}
$$

and we set

$$
g_{1}\left(x_{1}, \ldots, x_{m}, v_{1}^{(1)}, z_{1}^{(1)}, \ldots, v_{m}^{(1)}, z_{m}^{(1)}, Y\right)=\frac{1}{m !} \sum_{\sigma, \tau \in S_{m}}(\operatorname{sgn} \sigma)(\operatorname{sgn} \tau) g_{\sigma, \tau} .
$$


Clearly the polynomial $g_{1}$ is alternating on each of the sets $\left\{x_{1}, \ldots, x_{m}\right\},\left\{v_{1}^{(1)}, \ldots\right.$, $\left.v_{m}^{(1)}\right\}$ and $\left\{z_{1}^{(1)}, \ldots, z_{m}^{(1)}\right\}$. Next we show that $\varphi\left(g_{1}\right)=\operatorname{det} \bar{\beta}_{1} \varphi(f)$, for any evaluation $\varphi$.

Now, by Lemma 3 it is readily seen that

$$
\varphi\left(g_{1}\right)=\gamma \varphi(f)
$$

for any evaluation $\varphi: X \rightarrow L^{(+)}$, where

$$
\gamma=\frac{1}{m !} \sum_{\sigma, \tau \in S_{m}}(\operatorname{sgn} \sigma)(\operatorname{sgn} \tau) \beta\left(\bar{v}_{\sigma(1)}^{(1)}, \bar{z}_{\tau(1)}^{(1)}\right) \cdots \beta\left(\bar{v}_{\sigma(m)}^{(1)}, \bar{z}_{\tau(m)}^{(1)}\right) .
$$

We fix $\sigma \in S_{m}$ and we compute the sum

$$
\gamma_{\sigma}=\sum_{\tau \in S_{m}}(\operatorname{sgn} \tau) \beta\left(\bar{v}_{\sigma(1)}^{(1)}, \bar{z}_{\tau(1)}^{(1)}\right) \cdots \beta\left(\bar{v}_{\sigma(m)}^{(1)}, \bar{z}_{\tau(m)}^{(1)}\right) .
$$

In order to simplify the notation, write $\bar{v}_{\sigma(i)}^{(1)}=a_{i}, \bar{z}_{i}^{(1)}=b_{i}, i=1, \ldots, m$. Then

$$
\begin{aligned}
\gamma_{\sigma} & =\sum_{\tau \in S_{m}}(\operatorname{sgn} \tau) \beta\left(a_{1}, b_{\tau(1)}\right) \cdots \beta\left(a_{m}, b_{\tau(m)}\right)=\operatorname{det}\left(\begin{array}{ccc}
\beta\left(a_{1}, b_{1}\right) & \cdots & \beta\left(a_{1}, b_{m}\right) \\
\vdots & & \vdots \\
\beta\left(a_{m}, b_{1}\right) & \cdots & \beta\left(a_{m}, b_{m}\right)
\end{array}\right) \\
& =\left(\begin{array}{ccc}
\beta\left(a_{\sigma^{-1}(1)}, b_{1}\right) & \cdots & \beta\left(a_{\sigma^{-1}(1)}, b_{m}\right) \\
\vdots & & \vdots \\
\beta\left(a_{\sigma^{-1}(m)}, b_{1}\right) & \cdots & \beta\left(a_{\sigma^{-1}(m)}, b_{m}\right)
\end{array}\right)=(\operatorname{sgn} \sigma) \operatorname{det} \bar{\beta}_{1} .
\end{aligned}
$$

Hence

$$
\gamma=\frac{1}{m !} \sum_{\sigma \in S_{m}}(\operatorname{sgn} \sigma) \gamma_{\sigma}=\operatorname{det} \bar{\beta}_{1},
$$

and the proof is completed in case $k=1$.

If $k>1$, by the inductive hypothesis there exists a multilinear Lie polynomial

$$
g_{k-1}\left(x_{1}, \ldots, x_{m}, v_{1}^{(1)}, z_{1}^{(1)}, \ldots, v_{m}^{(1)}, z_{m}^{(1)}, \ldots, v_{1}^{(k-1)}, z_{1}^{(k-1)}, \ldots, v_{m}^{(k-1)}, z_{m}^{(k-1)}, Y\right)
$$

satisfying the conclusion of the lemma. We then write

$$
g_{k-1}=g_{k-1}\left(x_{1}, \ldots, \ldots, x_{m}, Y^{\prime}\right),
$$

where $Y^{\prime}=Y \cup\left\{v_{1}^{(1)}, z_{1}^{(1)}, \ldots, v_{m}^{(1)}, z_{m}^{(1)}, \ldots, v_{1}^{(k-1)}, z_{1}^{(k-1)}, \ldots, v_{m}^{(k-1)}, z_{m}^{(k-1)}\right\}$ and we apply to $g_{k-1}$, Lemma 3 and the previous arguments. In this way we can construct the polynomial $g_{k}$ and, for any evaluation $\varphi$, we have

$$
\varphi\left(g_{k}\right)=\operatorname{det} \bar{\beta}_{k} \varphi\left(g_{k-1}\right)=\operatorname{det} \bar{\beta}_{1} \cdots \operatorname{det} \bar{\beta}_{k} \varphi(f) .
$$

This completes the proof of the lemma.

\section{Multialternating Jordan polynomials}

Recall that $R=M_{q}(F), q \geq 3, L=s l_{q}(F), L^{(+)}=s l_{q}(F) \cap R^{(+)}$and $L^{(-)}=$ $R^{(-)}$. By making use of the results of the previous section, we can now construct multialternating Lie polynomials nonvanishing in $L^{(+)}$.

Lemma 6. For any $k \geq 0$ there exists a multilinear Lie polynomial

$$
g=g\left(x_{1}^{(1)}, \ldots, x_{m}^{(1)}, \ldots, x_{1}^{(2 k+1)}, \ldots, x_{m}^{(2 k+1)}, y_{1}, \ldots, y_{N}\right)
$$


satisfying the following conditions:

1) $g$ is alternating on each set $\left\{x_{1}^{(i)}, \ldots, x_{m}^{(i)}\right\}, 1 \leq i \leq 2 k+1$;

2) $g$ is not an identity of $L^{(+)}$;

3) the integer $N$ does not depend on $k$.

Proof. We start with the case $k=0$ by constructing a multilinear Lie polynomial $f=f\left(x_{1}, \ldots, x_{m}, y_{1}, \ldots, y_{N}\right)$ alternating on $x_{1}, \ldots, x_{m}$ and nonvanishing on $L^{(+)}$.

By a result of Razmyslov ([20, Theorem 12.1]) applied to the adjoint representation of the Lie algebra $L=s l_{q}(F)$, there exist an integer $r \geq 1$ and a multilinear polynomial $h\left(z_{1}, \ldots, z_{d r}, y\right)$, where $d=\operatorname{dim} L=q^{2}-1$, with the following properties: 1) $h$ is alternating on each set $\left.\left\{z_{(i-1) d+1}, \ldots, z_{i d}\right\}, 1 \leq i \leq r ; 2\right)$ for any evaluation $z_{i} \rightarrow \bar{z}_{i} \in L, y \rightarrow \bar{y} \in L$ we have that $h\left(\bar{z}_{1}, \ldots, \bar{z}_{d r}, \bar{y}\right)=\lambda \bar{y}$, for some $\lambda \in F ; 3) h$ is not an identity of $L$.

Let $\varphi: z_{i} \rightarrow \bar{z}_{i} \in L, y \rightarrow \bar{y} \in L$ be an evaluation such that $\varphi(h) \neq 0$. Then, for any $i=1, \ldots, r$, the elements $\bar{z}_{(i-1) d+1}, \ldots, \bar{z}_{i d}$ must be linearly independent over $F$. Since $d=\operatorname{dim} L$, this says that precisely $m=\operatorname{dim} L^{(+)}$among them lie in $L^{(+)}$. After renaming the variables, we may write

$$
h=h\left(x_{1}, \ldots, x_{m}, z_{1}, \ldots, z_{d r-m}, y\right),
$$

$h$ is alternating on $x_{1}, \ldots, x_{m}$ and $\varphi(h) \neq 0$, for some evaluation $\varphi: x_{1} \rightarrow$ $\bar{x}_{1}, \ldots, x_{m} \rightarrow \bar{x}_{m}$ in $L^{(+)}$and of the remaining variables in $L^{(+)} \cup L^{(-)}$.

Now, notice that $\left[L^{(+)}, L^{(+)}\right] \oplus L^{(+)}$is a nonzero ideal of the simple Lie algebra $L=s l_{q}(F)$. Hence $\left[L^{(+)}, L^{(+)}\right]=L^{(-)}$. This implies that if $\varphi$ is a nonzero evaluation of $h$ such that, say, $\varphi: z_{1} \rightarrow \bar{z}_{1} \in L^{(-)}$, then the polynomial

$$
h^{\prime}=h\left(x_{1}, \ldots, x_{m},\left[y_{1}, y_{2}\right], z_{2}, \ldots, z_{d r-m}, y\right)
$$

has a nonzero evaluation in $L^{(+)} \cup L^{(-)}$such that $\bar{x}_{1}, \ldots, \bar{x}_{m}, \bar{y}_{1}, \bar{y}_{2} \in L^{(+)}$. Next we apply the same procedure to all the variables $z_{i}$ which are evaluated in $L^{(-)}$. Then, after renaming the variables, we obtain a polynomial $f=f\left(x_{1}, \ldots, x_{m}, y_{1}, \ldots, y_{N}\right)$ alternating on $x_{1}, \ldots, x_{m}$ and nonvanishing in $L^{(+)}$. This completes the case $k=0$.

If $k \geq 1$, we apply Lemma 5 to $f$ and we obtain a polynomial $g_{k}$ which is alternating on $2 k+1$ sets of variables each of order $m$. By (44), for any evaluation $\varphi$ in $L^{(+)}$,

$$
\varphi\left(g_{k}\right)=\left(\operatorname{det} \bar{\beta}_{1}\right) \cdots\left(\operatorname{det} \bar{\beta}_{k}\right) \varphi(f) .
$$

Since by Lemma $4 \beta$ is nondegenerate, by (3) $\operatorname{det} \bar{\beta}_{i} \neq 0$, for some evaluation $\bar{\beta}_{i}$. Hence, since $f$ is not an identity of $L^{(+)}$it also follows that the polynomial $g_{k}$ is not an identity of $L^{(+)}$. This completes the proof of the lemma.

Suppose that there exists an element $a \in L$ such that $a b=b a$ or $a b=-b a$ for all $b \in L^{(+)}$. In this case, $a$ commutes with all the elements of $\left[L^{(+)}, L^{(+)}\right]=L^{(-)}$; hence it generates a 1-dimensional module for the Lie algebra $L^{(-)}$, under the adjoint action of $L$. Recall that $L=s l_{q}(F)$, and it is well known that since $q \geq 3$, $L$ is a faithful $L^{(-)}$-module. It follows that $a=0$. We have proved the following.

Remark 2. If $a \in L$ is such that $a b=b a$ or $a b=-b a$ for all $b \in L^{(+)}$, then $a=0$. 
Now we are ready to prove the existence of Jordan multialternating polynomials.

Theorem 1. Let $F$ be a field of characteristic zero and let $R=M_{q}(F)$ be the algebra of $q \times q$ matrices with transpose or symplectic involution $*, q \geq 3$. Let $R^{(+)}=\left\{a \in R \mid a^{*}=a\right\}$ be its Jordan subalgebra of symmetric elements. If $m=\operatorname{dim} R^{(+)}$, then, for all $k \geq 1$, there exists a multilinear Jordan polynomial

$$
h=h\left(x_{1}^{(1)}, \ldots, x_{m}^{(1)}, \ldots, x_{1}^{(2 k+1)}, \ldots, x_{m}^{(2 k+1)}, y_{1}, \ldots, y_{N}\right)
$$

satisfying the following conditions:

1) $h$ is alternating on each set $\left\{x_{1}^{(i)}, \ldots, x_{m}^{(i)}\right\}, 1 \leq i \leq 2 k+1$;

2) $h$ is not an identity of $R^{(+)}$;

3) $N$ does not depend on $k$.

Proof. Recall that $L=s l_{q}(F), L^{(+)}=L \cap R^{(+)}, L^{(-)}=L \cap R^{(-)}=R^{(-)}$and $L=L^{(+)} \oplus L^{(-)}$, a direct sum of vector spaces.

Since $\operatorname{dim} L^{(+)}=\operatorname{dim} R^{(+)}-1=m-1$, by Lemma 6 there exists a Lie polynomial

$$
g=g\left(x_{1}^{(1)}, \ldots, x_{m-1}^{(1)}, \ldots, x_{1}^{(2 k+1)}, \ldots, x_{m-1}^{(2 k+1)}, y_{1}, \ldots, y_{N}\right)
$$

nonvanishing on $L^{(+)}$. If $\operatorname{deg} g$ is odd, then by Lemma $g$ is a Jordan polynomial. In case $\operatorname{deg} g$ is even, we replace $g$ with the polynomial

$$
g^{\prime}=\left[g\left(x_{1}^{(1)}, \ldots, x_{m-1}^{(2 k+1)}, y_{1}, \ldots, y_{N}\right), y_{N+1}\right] .
$$

Let $\varphi: X \rightarrow L^{(+)}$be an evaluation such that $\varphi(g) \neq 0$. Then by Remark 2 , $\left[\varphi(g), \bar{y}_{N+1}\right] \neq 0$ for some $\bar{y}_{N+1} \in L^{(+)}$. This proves that $g^{\prime}$ does not vanish on $L^{(+)}$. Moreover $g^{\prime}$ is a Lie polynomial of odd degree. Hence, without loss of generality, we may assume that $g$ itself has odd degree.

Now define

$$
\begin{aligned}
h & =h\left(x_{1}^{(1)}, \ldots, x_{m}^{(1)}, \ldots, x_{1}^{(2 k+1)}, \ldots, x_{m}^{(2 k+1)}, y_{1}, \ldots, y_{N}\right) \\
& =\operatorname{Alt}_{1} \cdots \operatorname{Alt}_{2 k+1}\left(g \circ x_{m}^{(1)} \circ \cdots \circ x_{m}^{(2 k+1)}\right),
\end{aligned}
$$

where $\mathrm{Alt}_{i}$ means alternation on the variables $x_{1}^{(i)}, \ldots, x_{m}^{(i)}$. For instance,

$$
\begin{aligned}
& \operatorname{Alt}_{1}\left(g \circ x_{m}^{(1)}\right) \\
& \quad=\sum_{\sigma \in S_{m}}(\operatorname{sgn} \sigma) g\left(x_{\sigma(1)}^{(1)}, \ldots, x_{\sigma(m-1)}^{(1)}, \ldots, x_{1}^{(2 k+1)}, \ldots, x_{m-1}^{(2 k+1)}, y_{1}, \ldots, y_{N}\right) \circ x_{\sigma(m)}^{(1)} .
\end{aligned}
$$

Then $h$ satisfies the conclusion 1) of the theorem, and we need only to prove that $h$ is not an identity of $R^{(+)}$.

Since $g$ is not an identity of $L^{(+)}$, there exists an evaluation $\varphi: x_{i}^{(j)} \rightarrow \bar{x}_{i}^{(j)} \in$ $L^{(+)}, y_{j} \rightarrow \bar{y}_{j} \in L^{(+)}$with $\varphi(g)=\bar{g} \neq 0$. Now we extend $\varphi$ to $x_{m}^{(1)}, \ldots, x_{m}^{(2 k+1)}$, by setting $\varphi: x_{m}^{(i)} \rightarrow \bar{x}_{m}^{(i)}=E$, for all $i=1, \ldots, 2 k+1$, where $E$ is the identity $q \times q$ matrix.

Recalling that $g$ is a Lie polynomial, we get that

$$
g\left(\ldots, \bar{x}_{\sigma(1)}^{(j)}, \ldots, \bar{x}_{\sigma(m-1)}^{(j)}, \ldots\right) \circ \cdots \circ \bar{x}_{\sigma(m)}^{(j)} \circ \cdots=0,
$$

as soon as $\sigma(m) \neq m$. Therefore, since

$$
\sum_{\sigma \in S_{m}, \sigma(m)=m}(\operatorname{sgn} \sigma) g\left(\ldots, \bar{x}_{\sigma(1)}^{(j)}, \ldots, x_{\sigma(m-1)}^{(j)}, \ldots\right)=(m-1) ! \bar{g}
$$


we obtain

$$
\varphi(h)=((m-1) !)^{2 k+1} \bar{g} \circ E \circ \cdots \circ E=2^{2 k+1}((m-1) !)^{2 k+1} \bar{g} \neq 0
$$

and the proof is complete.

\section{Central polynomials for simple Jordan algebras OF BILINEAR FORMS}

In this section we let $R=F \oplus V$ be a Jordan algebra defined by a symmetric bilinear form $\beta$ on a vector space $V$. Recall that the product is defined by $u v=$ $\beta(u, v) \cdot 1$, where 1 is the unit element of $F$, and it acts on $V$ as the identity linear transformation. In case $\beta$ is nondegenerate and $\operatorname{dim} V \geq 2$, then $R$ is simple.

Recall that a central polynomial for an algebra $A$ with 1 is a polynomial $f$ whose values are scalar multiples of 1 in $A$ and $f$ is not a polynomial identity of $A$.

Proposition 1. If $\operatorname{dim} V=n$, then

$$
\begin{gathered}
f=f\left(x_{1}, \ldots, x_{n+1}, y_{1}, \ldots, y_{n+1}\right) \\
=\sum_{\sigma \in S_{n+1}, \tau \in S_{n+1}}(\operatorname{sgn} \sigma \tau)\left(x_{\sigma(1)} \circ y_{\tau(1)}\right) \circ \cdots \circ\left(x_{\sigma(n+1)} \circ y_{\tau(n+1)}\right)
\end{gathered}
$$

is a central polynomial of $R$.

Proof. We start by proving that $f$ takes scalar values in $R$, i.e., values of the type $\lambda=\lambda 1 \in F$.

Let $\left\{e_{1}, \ldots, e_{n}\right\}$ be an orthogonal basis of $V$, that is, $\beta\left(e_{i}, e_{j}\right)=0$ if and only if $i \neq j$. If we set $e_{n+1}=1 \in F$, then $\left\{e_{1}, \ldots, e_{n+1}\right\}$ is a basis of $R$. Since $f$ is multilinear it is enough to check that

$$
f\left(e_{i_{1}}, \ldots, e_{i_{n+1}}, e_{j_{1}}, \ldots, e_{j_{n+1}}\right)
$$

is central in $R$, for all $1 \leq i_{1}, j_{1}, \ldots, i_{n+1}, j_{n+1} \leq n+1$. Now, if $\left\{i_{1}, \ldots, i_{n+1}\right\} \neq$ $\{1, \ldots, n+1\}$, then the elements $e_{i_{1}}, \ldots, e_{i_{n+1}}$ are linearly dependent and the value of $f$ in (6) is zero, since $f$ is alternating on $x_{1}, \ldots, x_{n+1}$. Hence $\left\{i_{1}, \ldots, i_{n+1}\right\}=$ $\{1, \ldots, n+1\}$. Similarly $\left\{j_{1}, \ldots, j_{n+1}\right\}=\{1, \ldots, n+1\}$ and the value of $f$ in (6) is a linear combination of monomials of the type

$$
a=\left(e_{k_{1}} \circ e_{l_{1}}\right) \circ \cdots \circ\left(e_{k_{n+1}} \circ e_{l_{n+1}}\right),
$$

where $\left\{k_{1}, \ldots, k_{n+1}\right\}=\left\{l_{1}, \ldots, l_{n+1}\right\}=\{1, \ldots, n+1\}$. Suppose that $i$ and $j$ are such that $k_{i}=l_{j}=n+1$. Then, in case $i=j$, all factors $e_{k_{\alpha}} \circ e_{l_{\beta}}$ in (77) are scalars and $a \in F$. If $i \neq j$, then $e_{k_{i}} \circ e_{l_{i}}=e_{l_{i}}, e_{k_{j}} \circ e_{l_{j}}=e_{k_{j}}$ and, since any other product of the type $e_{k_{\alpha}} \circ e_{l_{\beta}}$ with $k_{\alpha}, l_{\beta} \leq n$ gives a scalar, we get that $a=\lambda\left(e_{k_{j}} \circ e_{l_{i}}\right)=\lambda^{\prime} \in F$. We have proved that all values of $f$ are scalar multiples of 1 in $R$.

Next we prove that $f$ is not an identity of $R$. We consider the evaluation $\varphi$ of $f$ such that $\varphi\left(x_{1}\right)=\varphi\left(y_{1}\right)=e_{1}, \ldots, \varphi\left(x_{n+1}\right)=\varphi\left(y_{n+1}\right)=e_{n+1}$. Then

$$
\varphi(f)=\sum_{\sigma \in S_{n+1}, \tau \in S_{n+1}}(\operatorname{sgn} \sigma \tau)\left(e_{\sigma(1)} \circ e_{\tau(1)}\right) \circ \cdots \circ\left(e_{\sigma(n+1)} \circ e_{\tau(n+1)}\right)=\sum_{\tau \in S_{n+1}} w_{\tau},
$$

where

$$
w_{\tau}=\sum_{\sigma \in S_{n+1}}(\operatorname{sgn} \sigma \tau)\left(e_{\sigma(1)} \circ e_{\tau(1)}\right) \circ \cdots \circ\left(e_{\sigma(n+1)} \circ e_{\tau(n+1)}\right)
$$


Now we fix $\tau \in S_{n+1}$ and we compute $w_{\tau}$. Since $S_{n+1}=S_{n+1} \tau$, we can write

$$
\begin{aligned}
w_{\tau}= & \sum_{\rho \in S_{n+1}}(\operatorname{sgn} \rho \tau \tau)\left(e_{\rho \tau(1)} \circ e_{\tau(1)}\right) \circ \cdots \circ\left(e_{\rho \tau(n+1)} \circ e_{\tau(n+1)}\right) \\
= & \sum_{\rho \in S_{n+1}}(\operatorname{sgn} \rho)\left(e_{\rho\left(i_{1}\right)} \circ e_{i_{1}}\right) \circ \cdots \circ\left(e_{\rho\left(i_{n+1}\right)} \circ e_{i_{(n+1)}}\right),
\end{aligned}
$$

where we have set $\tau(1)=i_{1}, \ldots, \tau(n+1)=i_{n+1}$.

If in (8), $\rho$ is such that $\rho(n+1)=n+1$, then the product $\left(e_{\rho\left(i_{1}\right)} \circ e_{i_{1}}\right) \circ \cdots \circ$ $\left(e_{\rho\left(i_{n+1}\right)} \circ e_{i_{n+1}}\right)=v_{\rho}$ is nonzero only if $\rho=(1)$. In this case $\operatorname{sgn} \rho=1$ and $v_{\rho}=\lambda \in F$, where $\lambda=\lambda_{1} \cdots \lambda_{n}$ with $\lambda_{i}=e_{i} \circ e_{i} \neq 0,1 \leq i \leq n$. Now let $\rho$ be such that $\rho(n+1)=t \neq n+1$. Then the factor $e_{\rho(t)} \circ e_{t}$ is nonzero only if $e_{\rho(t)}=e_{n+1}=1$, i.e., $\rho(t)=n+1$. In this case $v_{\rho}$ is nonzero only if $\rho(j)=j$, for all $j \neq t, n+1$. Hence $\rho=(t, n+1), v_{\rho}=\lambda \in F$ and $\operatorname{sgn} \rho=-1$.

It follows that $w_{\tau}=(1-n) \lambda \in F$. Therefore

$$
\varphi(f)=\sum_{\tau \in S_{n+1}} w_{\tau}=(n+1) !(1-n) \cdot \lambda \neq 0,
$$

and $f$ is not an identity of $R$.

\section{Codimension GROWTH}

In this section we shall compute the exponential rate of growth of the codimensions of any finite dimensional special simple Jordan algebra. We start by computing an upper bound of the codimensions of any finite dimensional algebra.

Let $F\{X\}$ be the free nonassociative algebra on a countable set $X$ and, for $n \geq 1$ let $P_{n}$ denote the space of multilinear polynomials of $F\{X\}$ in the variables $x_{1}, \ldots, x_{n}$. If $A$ is a not necessarily associative algebra, we still denote by $\operatorname{Id}(A)$ the $T$-ideal of $F\{X\}$ of polynomial identities of $A$ and $c_{n}(A)=\operatorname{dim} \frac{P_{n}}{P_{n} \cap \operatorname{Id}(A)}$.

Proposition 2. If $A$ is a finite dimensional algebra, $\operatorname{dim} A=d$, then $c_{n}(A) \leq d^{n+1}$.

Proof. Let $a_{1}, \ldots, a_{d}$ be a basis of $A$ over $F$. For a fixed $n$, let

$$
m_{1}\left(x_{1}, \ldots, x_{n}\right), \ldots, m_{N}\left(x_{1}, \ldots, x_{n}\right)
$$

be a basis of $P_{n}$ over $F$. Then a multilinear polynomial

$$
f\left(x_{1}, \ldots, x_{n}\right)=\sum \alpha_{j} m_{j}\left(x_{1}, \ldots, x_{n}\right)
$$

is an identity of $A$ if and only if it vanishes under any evaluation $x_{1} \rightarrow a_{i_{1}}, \ldots, x_{n} \rightarrow$ $a_{i_{n}}$ i.e., the following linear relation is satisfied:

$$
f\left(a_{i_{1}}, \ldots, a_{i_{n}}\right)=\sum \alpha_{j} m_{j}\left(a_{i_{1}}, \ldots, a_{i_{n}}\right)=0 .
$$

The total number of such evaluations is $d^{n}$ since any $x_{i}$ can take $d$ distinct values. We regard the coefficients $\alpha_{j}$ as indeterminates. By rewriting the right-hand side of (9) as a linear combination of the basis elements of $A$, any relation (9) can be viewed as $d$ linear equations on the $\alpha_{j}$ 's since $\operatorname{dim} A=d$. Hence, (9) can be regarded as a system of $d^{n+1}$ linear equations in $N$ indeterminates $\alpha_{j}, 1 \leq j \leq N$. The space of solutions has dimension $N-r$, where $r$ is the rank of the system, $r \leq d^{n+1}$. Any solution of (9), i.e., any $N$-tuple of coefficients $\alpha_{j}, 1 \leq j \leq N$, gives a multilinear identity of $A$. Moreover, linearly independent solutions give linearly independent 
identities. Therefore $\operatorname{dim}\left(P_{n} \cap I d(A)\right)=N-r$ and $c_{n}(A)=\operatorname{dim} P_{n} /\left(P_{n} \cap I d(A)\right)=$ $r \leq d^{n+1}$.

Now we restrict our attention to Jordan algebras. Let $P_{n}^{J}$ be the space of multilinear Jordan polynomials in $x_{1}, \ldots, x_{n}$. Recall that $P_{n}^{J}$ is spanned by the Jordan products $x_{\sigma(1)} \circ x_{\sigma(2)} \circ \cdots \circ x_{\sigma(n)}$, where $\sigma \in S_{n}$ with all possible arrangements of brackets. Given a Jordan algebra $B$ denote by $I d^{J}(B)$ the $T$-ideal of $F^{J}\langle X\rangle$ of polynomial identities of $B$.

It is well known that the symmetric group $S_{n}$ acts on $P_{n}^{J}$ : if $\sigma \in S_{n}$ and $f\left(x_{1}, \ldots, x_{n}\right) \in P_{n}^{J}$, then $\sigma f\left(x_{1}, \ldots, x_{n}\right)=f\left(x_{\sigma(1)}, \ldots, x_{\sigma(n)}\right)$. We refer the reader to [15] for a description of the representation theory of $S_{n}$. As in the associative case, if $B$ is a Jordan algebra, $P_{n}^{J} \cap I d^{J}(B)$ is an $S_{n}$-module and $c_{n}^{J}(B)=\operatorname{dim} \frac{P_{n}^{J}}{P_{n}^{J} \cap I d^{J}(A)}$ is called the $n$th Jordan codimension of $B$. In case $B$ is a Jordan algebra such that its codimensions $c_{n}^{J}(B), n=1,2, \ldots$, are exponentially bounded (e.g., if $B$ is finite dimensional), one defines the Jordan exponent of $B$ as $\exp ^{J}(B)=\lim _{n \rightarrow \infty} \sqrt[n]{c_{n}^{J}(B)}$, in case such a limit exists.

In the next theorem we prove that for most special simple Jordan algebras $B$, the Jordan exponent exists and $\exp ^{J}(B)=\operatorname{dim} B$.

Theorem 2. Let $F$ be a field of characteristic zero, $R=M_{q}(F), q \geq 3$, with an involution $*$ and $R^{(+)}$its Jordan subalgebra of symmetric elements. Then, for all $n \geq 1$, there exist constants $C>0, t$ such that

$$
C n^{t} m^{n} \leq c_{n}^{J}\left(R^{(+)}\right) \leq m^{n+1},
$$

where $m=\operatorname{dim} R^{(+)}$. Hence the Jordan exponent of $R^{(+)}$exists and $\exp ^{J}\left(R^{(+)}\right)=$ $\operatorname{dim} R^{(+)}$.

Proof. It is well known that the codimensions of an algebra do not change upon extension of the base field. Hence without loss of generality we may assume that $F$ is algebraically closed and $*$ is either the transpose or the symplectic involution (see for instance the proof of [11, Theorem 3.6.8]).

By Theorem 1, for all $k \geq 1$, there exists a multilinear Jordan polynomial

$$
h=h\left(x_{1}^{(1)}, \ldots, x_{m}^{(1)}, \ldots, x_{1}^{(2 k+1)}, \ldots, x_{m}^{(2 k+1)}, y_{1}, \ldots, y_{N}\right)
$$

such that $h$ is alternating on each set of indeterminates $\left\{x_{1}^{(i)}, \ldots, x_{m}^{(i)}\right\}, 1 \leq i \leq$ $2 k+1$, and $h$ is not a polynomial identity of $R^{(+)}$. For simplicity we rename the variables and we write

where $Y=\left\{y_{1}, \ldots, y_{N}\right\}$.

$$
h=h\left(x_{1}, \ldots, x_{(2 k+1) m}, Y\right),
$$

For a fixed $k$, let $n=(2 k+1) m$. By abuse of notation, we let $P_{n+N}^{J}$ be the space of multilinear Jordan polynomials in $x_{1}, \ldots, x_{n}, y_{1}, \ldots, y_{N}$. Then $h \in P_{n+N}^{J}$ and we regard $P_{n+N}^{J}$ as an $S_{n}$-module by letting $S_{n}$ act on $x_{1}, \ldots, x_{n}$. We then consider $F S_{n} h$, the $S_{n}$-submodule of $P_{n+N}^{J}$ generated by $h$, and we decompose it into irreducible submodules.

Let $\lambda \vdash n$ be a partition of $n$. Given a Young tableau $T_{\lambda}$ of shape $\lambda \vdash n$, let $R_{T_{\lambda}}$ and $C_{T_{\lambda}}$ denote the subgroups of $S_{n}$ stabilizing the rows and the columns of $T_{\lambda}$, respectively. It is well known that if we let

$$
\bar{R}_{T_{\lambda}}=\sum_{\sigma \in R_{T_{\lambda}}} \sigma \text { and } \quad \bar{C}_{T_{\lambda}}=\sum_{\tau \in C_{T_{\lambda}}}(\operatorname{sgn} \tau) \tau
$$


the element $e_{T_{\lambda}}=\bar{R}_{T_{\lambda}} \bar{C}_{T_{\lambda}}$ is an essential idempotent of the group algebra $F S_{n}$, generating an irreducible $S_{n}$-module associated to $\lambda$.

Since $h \notin I d^{J}\left(R^{(+)}\right)$, there exists a Young tableau $T_{\lambda}, \lambda \vdash n$, such that $e_{T_{\lambda}} h \not \equiv 0$ in $R^{(+)}$. Write $\lambda=\left(\lambda_{1}, \ldots, \lambda_{r}\right)$.

If $\lambda_{1} \geq 2 k+2$, then $e_{T_{\lambda}} h$ is symmetric on at least $2 k+2$ variables among $x_{1}, \ldots, x_{n}$. But for any $\sigma \in \bar{R}_{T_{\lambda}}$ these variables in $\sigma \bar{C}_{T_{\lambda}}$ are divided into $2 k+1$ disjoint alternating subsets. It follows that $\sigma \bar{C}_{T_{\lambda}} h$ is alternating and symmetric on at least two variables and, so, $e_{T_{\lambda}} h=0$ in the zero polynomial, a contradiction. Thus $\lambda_{1} \leq 2 k+1$.

Suppose now that $r \geq m+1$. Since the first column of $T_{\lambda}$ is of height at least $m+1$, the polynomial $\bar{C}_{T_{\lambda}} h$ is alternating on at least $m+1$ variables among $x_{1}, \ldots, x_{n}$. From $\operatorname{dim} R^{(+)}=m$ it follows that for any $\sigma, \sigma \bar{C}_{T_{\lambda}} h \equiv 0$ on $R^{(+)}$and, so, also $e_{T_{\lambda}} h=\bar{R}_{T_{\lambda}} \bar{C}_{T_{\lambda}} h \equiv 0$ on $R^{(+)}$, a contradiction.

We have proved that $F S_{n} e_{T_{\lambda}} h \nsubseteq I I d^{J}\left(R^{(+)}\right)$, for some Young tableau $T_{\lambda}$ of shape $\lambda=\left((2 k+1)^{m}\right)$, a rectangle of width $2 k+1$ and height $m$.

Now let $n \geq m+N$ be an arbitrary integer and write $n=(2 k+1) m+N+r$, for some $k \geq 1$ and $0 \leq r \leq 2 m$. Let $h=h\left(x_{1}, \ldots, x_{(2 k+1) m}, Y\right)$ be the polynomial constructed above and set $h^{\prime}=h$, if $r=0$, or

$h^{\prime}\left(x_{1}, \ldots, x_{(2 k+1) m+r}, Y\right)=h\left(x_{1}, \ldots, x_{(2 k+1) m}, Y\right) \circ x_{(2 k+1) m+1} \circ \cdots \circ x_{(2 k+1) m+r}$,

if $r>0$. Clearly $h^{\prime} \in P_{n}^{J}$. Moreover, if $\lambda=\left((2 k+1)^{m}\right)$ and $T_{\lambda}$ is the Young tableau given above such that $e_{T_{\lambda}} h \notin I d^{J}\left(R^{(+)}\right)$, it is clear that $e_{T_{\lambda}} h^{\prime} \notin I d^{J}\left(R^{(+)}\right)$ also holds.

Write $F S_{n}=\bigoplus_{\mu \vdash n} I_{\mu}$, where $I_{\mu}$ is the minimal two-sided ideal of $F S_{n}$ corresponding to the partition $\mu$. By the branching rule of $S_{(2 k+1) m}$ (see 15, Theorem 2.4.3]) we have that

$$
F S_{n} e_{T_{\lambda}} h^{\prime} \subseteq \bigoplus_{\substack{\mu \supset \lambda \\ \mu \vdash n}} I_{\mu} h^{\prime},
$$

and, since $e_{T_{\lambda}} h^{\prime} \notin I d^{J}\left(R^{(+)}\right)$, there exists a partition $\mu \vdash n$ and a tableau $T_{\mu}$ such that $F S_{n} e_{T_{\mu}} h^{\prime} \nsubseteq I d^{J}\left(R^{(+)}\right)$. This says that $c_{n}^{J}\left(R^{(+)}\right) \geq d_{\mu}=\operatorname{dim} F S_{n} e_{T_{\mu}}$. But again by the branching rule, $d_{\mu} \geq d_{\lambda}=d_{\left((2 k+1)^{m}\right)}$. Since $n-(2 k+1) m \leq r$ and asymptotically $d_{\left((2 k+1)^{m}\right)} \simeq C_{0}((2 k+1) m)^{s} m^{(2 k+1) m}$, for some constants $C_{0}, s$ (see [11, Lemma 6.2.5]), we obtain that

$$
c_{n}^{J}\left(R^{(+)}\right) \geq C n^{t} m^{n},
$$

for some constants $C>0, t$.

Putting together Proposition 2 and the above inequality, we get $C n^{t} m^{n} \leq$ $c_{n}^{J}\left(R^{(+)}\right) \leq m^{n+1}$, for some constants $C$, t. In particular $\lim _{n \rightarrow \infty} \sqrt[n]{c_{n}^{J}\left(R^{(+)}\right)}=m$, and we are done.

The same result also holds for Jordan algebras of bilinear forms.

Theorem 3. Let $R$ be a finite dimensional simple Jordan algebra of a bilinear form over a field of characteristic zero. Then, for all $n \geq 1$, there exist constants $C>0, t$ such that

$$
C n^{t} m^{n} \leq c_{n}^{J}(R) \leq m^{n+1},
$$

where $m=\operatorname{dim} R$. Hence the Jordan exponent of $R$ exists and $\exp ^{J}(R)=\operatorname{dim} R$. 
Proof. By Proposition 1 there exisits a multilinear polynomial $f\left(x_{1}, \ldots, x_{m}, y_{1}, \ldots\right.$, $\left.y_{m}\right)$ alternating on $x_{1}, \ldots, x_{m}$ and on $y_{1}, \ldots, y_{m}$, where $m=\operatorname{dim} R$, which takes scalar values on $R$ and is not an identity of $R$. Hence, for all $k \geq 1$ there exists a multilinear Jordan polynomial

$$
h=h\left(x_{1}^{(1)}, \ldots, x_{m}^{(1)}, \ldots, x_{1}^{(2 k)}, \ldots, x_{m}^{(2 k)}\right)
$$

such that $h$ is alternating on each set of indeterminates $\left\{x_{1}^{(i)}, \ldots, x_{m}^{(i)}\right\}, 1 \leq i \leq 2 k$, and $h$ is not a polynomial identity of $R$. The same arguments as in the previous theorem give us a lower bound for the codimensions:

$$
c_{n}^{J}(R) \geq C n^{t} m^{n}
$$

for some constants $C>0, t$.

By Proposition 2 we get $c_{n}^{J}(B) \leq m^{n+1}$. Hence $\lim _{n \rightarrow \infty} \sqrt[n]{c_{n}^{J}\left(R^{(+)}\right)}=m$ and the proof is complete.

We can now state the following result.

Theorem 4. Let $R$ be a finite dimensional special simple Jordan algebra over a field of characteristic zero. Then $\exp ^{J}(R)$ exists and equals the dimension of $R$ over its center.

Proof. Since the codimensions do not change upon extension of the base field, we may regard $R$ as an algebra over its center $F$. Now, if $\bar{F}$ is the algebraic closure of $F$, then $\bar{R}=R \otimes_{F} \bar{F}$ is a special simple Jordan algebra over $\bar{F}$ and $\operatorname{dim}_{F} R=\operatorname{dim}_{\bar{F}} \bar{R}$. Putting together [14, Corollary 2, p. 204], Theorem 2 and Theorem 3, we get the desired conclusion.

Corollary 1. Let $R$ be a finite dimensional special semisimple Jordan algebra over a field of characteristic zero. Then $\exp ^{J}(R)$ exists and is an integer.

Proof. Write $R=R_{1} \oplus \cdots \oplus R_{m}$, a direct sum of special simple Jordan algebras. Since for all $1 \leq i \leq m, c_{n}\left(R_{i}\right) \leq c_{n}(R) \leq c_{n}\left(R_{1}\right)+\cdots+c_{n}\left(R_{m}\right)$, we get that $\exp ^{J}(R)=\max _{i}\left(\exp ^{J}\left(R_{i}\right)\right)$.

\section{LOWER BOUNDS FOR THE CODIMENSION GROWTH}

In this section we want to determine the least exponential rate of growth of a finite dimensional Jordan algebra. In general, for nonassociative algebras, in 5 , Theorem 2] it was proved that for a finite dimensional algebra $A, \operatorname{dim} A=d, c_{n}(A)$ is either polynomially bounded or $c_{n}(A)>\frac{1}{n^{2}} 2^{\frac{n}{d^{2}}}$ for $n$ large enough. Moreover, for any $1<\alpha<\beta<2$, in [7] the authors constructed a finite dimensional algebra $B$ such that $\alpha<\exp (B)<\beta$. In the next theorem we show that, as in the case of associative or Lie algebras, finite dimensional special Jordan algebras cannot have exponential rate of growth of the codimensions strictly between 1 and 2 .

Theorem 5. Let $R$ be a finite dimensional special Jordan algebra. Then either the sequence of codimensions $c_{n}^{J}(R)$ is polynomially bounded or $c_{n}^{J}(R) \geq C n^{q} 2^{n}$ for some constants $C>0, q$.

Proof. As we remarked in the previous section, we may assume that $F$ is algebraically closed and let us denote by $I$ the nilpotent radical of $R$. If $I=R$, then $R$ 
is nilpotent and $c_{n}^{J}(R)=0$ asymptotically. Hence we may assume that $I \neq R$. By [25, p. 97 Corollary] $A / I$ is also a special Jordan algebra and let

$$
R / I=B_{1} \oplus \cdots \oplus B_{m}
$$

be the decomposition of $A / I$ into the sum of simple algebras. If there exists $j \geq 1$ such that $\operatorname{dim} B_{j}=d>1$, then by Theorem 3 we have that $c_{n}^{J}(A) \geq c_{n}^{J}(A / I) \geq$ $c_{n}^{J}\left(B_{j}\right)=C n^{t} d^{n}$ and we are done.

Hence we may assume that $B_{1} \cong \ldots \cong B_{m} \cong F$ (as Jordan algebras). Since $I$ is nilpotent, by [14. Lemma 3, p. 149] any set of orthogonal idempotents can be lifted modulo $I$. Hence, since $B_{1}, \ldots, B_{m}$ are unitary algebras, there exist $m$ nonzero orthogonal idempotents $e_{1}, \ldots, e_{m}$ in $R$.

Recall that

$$
c_{n}^{J}(R)=\operatorname{dim} \frac{P_{n}^{J}}{P_{n}^{J} \cap I d(R)}
$$

(see Section 5) and consider the structure of an $S_{n}$-module on $M=\frac{P_{n}^{J}}{P_{n}^{J} \cap I d(R)}$. By complete reducibility $M$ decomposes into a finite sum of irreducible submodules and by [5, Theorem 1] the number of such submodules is a polynomially bounded function of $n$. Therefore in order to complete the proof we need only to estimate the dimension of an irreducible submodule of $M$.

First suppose that any multilinear Jordan monomial $m=m\left(x_{1}, \ldots, x_{n}\right)$ vanishes in $R$ provided that two variables are evaluated in $e_{i}$ and $e_{j}$, for some $i \neq j$. That is,

$$
m(\underbrace{e_{1}, \ldots, e_{1}}_{p_{1}}, \ldots, \underbrace{e_{m}, \ldots, e_{m}}_{p_{m}}, a_{1}, \ldots, a_{k})
$$

is equal to 0 in $R$ as soon as at least two among $p_{1}, \ldots, p_{m}$ are nonzero, $a_{1}, \ldots, a_{k} \in$ $I, k \geq 0$, and $p_{1}+\cdots+p_{m}+k=n$. We shall prove that in this case, $c_{n}^{J}(R)$ is polynomially bounded.

Now we consider the embedding of $A$ into its special universal envelope $U$. Then $I \subseteq \operatorname{rad}(U)$ where $\operatorname{rad}(U)$ is the Jacobson radical of $U$.

Let $\lambda=\left(\lambda_{1}, \ldots, \lambda_{r}\right) \vdash n$ be such that $\lambda_{2}+\cdots+\lambda_{r} \geq N$ where $\operatorname{rad}(U)^{N}=0$. For any multilinear polynomial $h=h\left(x_{1}, \ldots, x_{n}\right)$, the polynomial $\bar{C}_{T_{\lambda}} h$ contains $\lambda_{2}$ sets of alternating variables, each of order at least 2 . When evaluating the variables in $R$, we must substitute either at least two distinct $e_{i}$ 's or at least two equal $e_{i}$ 's in an alternating set or at least $N$ elements from $I$. In all cases the value of the polynomial is zero. It follows that

$$
F S_{n} e_{T_{\lambda}} h \not \equiv 0
$$

only if $\lambda_{2}+\cdots+\lambda_{r}<N$. From the hook formula that gives the dimension of an irreducible $F S_{n}$-module (see [15]), it easily follows that $\operatorname{dim} F S_{n} e_{T_{\lambda}}$ is bounded by a polynomial of degree $N$ and the proof is complete in this case.

Now suppose that there exists a monomial $m\left(x_{1}, \ldots, x_{n}\right)$ with a nonzero evaluation (11), where at least two among $p_{1}, \ldots, p_{m}$ are nonzero and $a_{1}, \ldots, a_{k} \in I$. In order to simplify the notation suppose $p_{1} \neq 0, p_{2} \neq 0$ and rename the variable so that $m=m\left(x_{1}, x_{2}, y_{1}, \ldots, y_{t}\right)$ and $m\left(e_{1}, e_{2}, \bar{y}_{1}, \ldots, \bar{y}_{t}\right) \neq 0$, for some $\bar{y}_{1}, \ldots, \bar{y}_{t} \in R$. 
Starting from $m$ we shall now construct a polynomial in $n$ pairs of alternating variables, for all $n=1,2, \ldots$. In fact define

$$
\begin{aligned}
f & =f\left(x_{1}, \ldots, x_{2 n+2}, y_{1}, \ldots, y_{t}\right) \\
& =\sum_{\sigma_{1}, \ldots, \sigma_{n} \in S_{2}}\left(\operatorname{sgn} \sigma_{1}\right) \cdots\left(\operatorname{sgn} \sigma_{n}\right) \\
& \times m\left(x_{\sigma_{1}(1)} \cdots x_{\sigma_{n}(n)} x_{2 n+1}, x_{\sigma_{1}(n+1)} \cdots x_{\sigma_{n}(2 n)} x_{2 n+2}, y_{1}, \ldots, y_{t}\right),
\end{aligned}
$$

where $\sigma_{i} \in S_{2}$ acts on the indices $i, n+i$. By evaluating

$$
\begin{array}{ll}
x_{1}, \ldots, x_{n}, x_{2 n+1} \rightarrow e_{1}, & x_{n+1}, \ldots, x_{2 n}, x_{2 n+2} \rightarrow e_{2}, \\
y_{1} \rightarrow \bar{y}_{1}, \ldots, y_{t} \rightarrow \bar{y}_{t}, &
\end{array}
$$

we get a sum of $2^{n}$ evaluated monomials corresponding to the distinct $n$-tuples of permutations $\left(\sigma_{i_{1}}, \ldots, \sigma_{i_{n}}\right)$. If at least one $\sigma_{i_{j}}$ is different from 1 , then the value of the corresponding summand is zero since $e_{1} e_{2}=0$. Hence the only nonzero summand is $m\left(e_{1}^{n+1}, e_{2}^{n+1}, \bar{y}_{1}, \ldots, \bar{y}_{t}\right)=m\left(e_{1}, e_{2}, \bar{y}_{1}, \ldots, \bar{y}_{t}\right) \neq 0$ and $f$ is not an identity of $R$. On the other hand, if we let $S_{2 n}$ act on $P_{2 n+t+2}^{J}$ by the permutation action only on the variables $x_{1}, \ldots, x_{2 n}$, the complete linearization of $f$ generates in $P_{2 n+t+2}^{J}$ an $S_{2 n}$-module whose irreducible components correspond to the partition $\lambda=(n, n)$. Now, by the hook formula, for any $\mu \vdash 2 n+t+2$ such that $\mu \geq \lambda$ in the left lexicographic order, we have that $d_{\mu} \geq d_{\lambda}$. Hence, since asymptotically $d_{\lambda} \simeq C_{0}(2 n)^{s} 2^{2 n}$ (see, for example [11, Lemma 6.2.5]), and $t+2$ is constant, one can easily find constants $C>0$ and $q$ such that $c_{n}^{J}(R) \geq C n^{q} 2^{n}$ for all $n$.

\section{REFERENCES}

[1] Bahturin, Yu. A.; Sehgal, S. K.; Zaicev, M. V., Group gradings on associative algebras, J. Algebra 241 (2001), 677-698. MR.1843319 (2002h:16067)

[2] Bahturin, Yu.; Zaicev, M., Group gradings on simple Lie algebras of type "A", J. Lie Theory 16 (2006), 719-742. MR2270657 (2007i:17037)

[3] Berele, A.; Regev, A., Hook Young diagrams with applications to combinatorics and to representations of Lie superalgebras, Adv. Math. 64 (1987), no. 2, 118-175. MR.884183(88i:20006)

[4] Berele, A.; Regev, A., Exponential growth for codimensions of some p.i. algebras, J. Algebra 241 (2001), 118-145. MR.1838847 (2002k:16046)

[5] Giambruno, A.; Mishchenko, S.; and Zaicev, M., Algebras with intermediate growth of the codimensions, Adv. in Appl. Math. 37 (2006), no. 3, 360-377. MR2261178 (2007g:16033)

[6] Giambruno, A.; Mishchenko, S.; and Zaicev, M., Codimension growth of two-dimensional non-associative algebras, Proc. Amer. Math. Soc. 135 (2007), no. 11, 3405-3415 MR 2336552 (2008f:17005)

[7] Giambruno, A.; Mishchenko, S.; Zaicev, M., Codimensions of algebras and growth functions, Adv. Math. 217 (2008), 1027-1052. MR2383893

[8] Giambruno, A.; Mishchenko, S.; and Zaicev, M., Polynomial identities of algebras of smal $l$ dimension, Comm. Algebra (to appear).

[9] Giambruno, A.; Regev, A.; Zaicev, M. V., Simple and semisimple Lie algebras and codimension growth, Trans. Amer. Math. Soc. 352 (2000), no. 4, 1935-1946. MR1637070 (2000i:17006)

[10] Giambruno, A.; Zaicev, M., Exponential codimension growth of P.I. algebras: An exact estimate, Adv. Math. 142 (1999), 221-243. MR1680198 (2000a:16048)

[11] Giambruno, A.; Zaicev, M., Polynomial Identities and Asymptotic Methods, Mathematical Surveys and Monographs Vol. 122, American Mathematical Society, Providence, RI, 2005. MR2176105 (2006g:16054)

[12] Giambruno, A.; Zaicev, M., Multialternating Jordan polynomials and codimension growth of matrix algebras, Linear Algebra Appl. 422 (2007), 372-379. MR 2305126 (2008c:17022)

[13] Goto, M.; Grosshans, F. D., Semisimple Lie algebras, Lecture Notes in Pure and Applied Mathematics, Vol. 38, Marcel Dekker, Inc., New York-Basel, 1978. MR0573070 (58:28084) 
[14] Jacobson, N., Structure and representations of Jordan algebras, American Mathematical Society Colloquium Publications, Vol. XXXIX, American Mathematical Society, Providence, R.I. 1968. MR0251099 (40:4330)

[15] James, G.; Kerber, A., The Representation Theory of the Symmetric Group, Encyclopedia of Mathematics and its Applications, Vol. 16, Addison-Wesley, London, 1981. MR644144 (83k:20003)

[16] Kemer, A. R. The Spechtian nature of $T$-ideals whose codimensions have power growth. (Russian) Sibirsk. Mat. Ž. 19 (1978), no. 1, 54-69; translation in Siberian Math. J., 19 (1978), 37-48. MR0466190(57:6070)

[17] Mishchenko, S. P., Growth of varieties of Lie algebras, (Russian) Uspekhi Mat. Nauk 45 (1990), 25-45, 189; translation in Russian Math. Surveys 45 (1990), 27-52. MR.1101331 (92g:17003)

[18] Mishchenko, S. P. Lower bounds on the dimensions of irreducible representations of symmetric groups and of the exponents of the exponential of varieties of Lie algebras. (Russian) Mat. Sb. 187 (1996), no. 1, 83-94; translation in Sb. Math. 187 (1996), no. 1, 81-92 MR.1380205 (97d:17003)

[19] Petrogradskiŭ, V. M., Growth of polynilpotent varieties of Lie algebras, and rapidly increasing entire functions, (Russian) Mat. Sb. 188 (1997), no. 6, 119-138; translation in Sb. Math. 188 (1997), no. 6, 913-931. MR:1479133 (99a:17008)

[20] Razmyslov, Yu. P., Identities of algebras and their representations, Translations of Mathematical Monographs, Vol. 138, American Mathematical Society, Providence, RI, 1994. MR.1291603 (95i:16022)

[21] Regev, A., Existence of identities in $A \otimes B$, Israel J. Math. 11 (1972), 131-152. MR0314893 $(47: 3442)$

[22] Zaicev, M. V., Varieties and identities of affine Kac-Moody algebras, Methods in ring theory, 303-314, Lecture Notes in Pure and Appl. Math., 198, Marcel Dekker, New York, 1998. MR:1767987 (2001j:17045)

[23] Zaicev, M., Integrality of exponents of growth of identities of finite-dimensional Lie algebras, (Russian), Izv. Ross. Akad. Nauk Ser. Mat. 66 (2002), 23-48; English translation: Izv. Math. 66 (2002), 463-487. MR1921808 (2003g:17004)

[24] Zaicev, M. V.; Mishchenko, S. P. The growth of varieties of Lie superalgebras with nilpotent commutator subalgebra, preprint.

[25] Zhevlakov, K. A.; Slin'ko, A. M.; Shestakov, I. P.; Shirshov, A. I. Rings that are nearly associative. Pure and Applied Mathematics, 104. Academic Press, Inc., New York-London, 1982. MR668355 (83i:17001)

Dipartimento di Matematica e Applicazioni, Università di Palermo, Via Archirafi 34, 90123 PALERmo, Italy

E-mail address: a.giambruno@unipa.it

Department of Algebra, Faculty of Mathematics and Mechanics, Moscow State UniVERSITY, Moscow, 119992 Russia

E-mail address: zaicev@mech.math.msu.su 